\title{
Two dimensional mapping of the perfusion of the retina and optic nerve head
}

\author{
G Michelson, B Schmauss
}

\begin{abstract}
Aim-To present a new non-invasive method of performing a high definition topography of perfused vessels of the retina and the optic nerve head with simultaneous evaluation of blood flow.
\end{abstract}

Method-By a combination of a laser Doppler flowmeter with a scanning laser system the perfusion of the retina and the optic nerve head is visualised. The principles of measuring blood flow by laser Doppler flowmetry are based on the optical Doppler effect: laser light scattered by a moving particle is shifted in frequency by an amount $\Delta f$. Our data acquisition and evaluation system is a modified laser scanning tomograph. The technical data are retinal area of measurement $2.7 \mathrm{~mm} \times 0.7 \mathrm{~mm}, 10^{\circ}$ field with 256 points $\times 64$ lines, measurement accuracy $10 \mu \mathrm{m}$, wavelength $670 \mathrm{~nm}$ and $790 \mathrm{~nm}$, light power $100 \mu \mathrm{W}$ and $200 \mu \mathrm{W}$, data acquisition time $2 \cdot 048 \mathrm{~s}$. Every line is scanned 128 times by a line sampling rate of $4000 \mathrm{~Hz}$. By performing a discrete fast Fourier transformation over 128 intensities of each retinal point the laser Doppler shift is calculated for each retinal point. With these data a two dimensional map with $256 \times 64$ points of the retinal perfusion is created. The brightness of the pixel is coded by the value of the Doppler shift. Offline capillary blood flow is estimated in arbitrary units according to the theory of laser Doppler flowmetry in every region of interest of the perfusion picture. We estimated the reliability and the validity of the method. Retinal blood flow was measured by scanning laser Doppler flowmetry (SLDF) while varying intraocular pressure by a suction cup of three healthy volunteers. Measurements of retinal blood flow performed in 47 eyes by the presented method (SLDF) were correlated with data gained by a commercially available laser Doppler flowmeter. Perfusion pictures of the superficial retinal layer and of deep prelaminar layers in the optic nerve head are presented.

Results-The reliability coefficients $r_{1}$ of 'flow', 'volume', and 'velocity' were 0.84 , 0.85 , and 0.84 respectively. We found a significant linear relation between SLDF flow and the ocular perfusion pressure $(r=0.84, p<0.001)$. Comparative measurements of the retinal blood flow by SLDF and a commercially available laser Doppler flowmeter showed a linear and significant relation (flow $r=0 \cdot 6, p<0.0001$, volume $r=0.4, p<0.01)$. Capillaries of the retinal superficial vasculature or deep ciliary sourced capillaries of the optic nerve head became visible with a high resolution by the confocal technique dependent on the focus. Offline, the blood flow variables of areas of $100 \mu \mathrm{m} \times 100 \mu \mathrm{m}$ were calculated.

Conclusion-SLDF enables the visualisation of perfused capillaries and vessels of the retina and the optic nerve head in high resolution by two dimensional mapping of perfusion variables which are encoded by the Doppler signal. This method achieves simultaneously qualitative and quantitative evaluation of capillary blood flow of distinct areas of the capillary meshwork.

(Br f Ophthalmol 1995; 79: 1126-1132)

Fluorescein angiography of the retina is the gold standard for the diagnosis of diseases of the retinal vasculature (for example, diabetic retinopathy) and shows vessels and capillaries of the retinal vasculature in a high definition. By combining a video system and/or a scanning laser ophthalmoscope it is possible to calculate the blood velocity and the arteriovenous passage time in the retinal vasculature. ${ }^{1}$ Fluorescein angiography is an invasive method and it is estimated that severe allergic reactions to fluorescein occur in $0.4 \%$ of all angiographies. ${ }^{2}$ Some authors have shown that single point measurement of the blood flow of the iris, retina, or optic nerve head can be achieved by laser Doppler flowmetry. ${ }^{34}$

We present a non-invasive method enabling a high resolution topography of perfused vessels of the retina and the optic nerve head with simultaneous evaluation of the blood flow variables.

\section{Method}

A method combining laser Doppler flowmetry and scanning laser technique known from laser scanning ophthalmoscopes is used to visualise and to characterise the perfusion of the retina and optic nerve head.

\section{LASER DOPPLER FLOWMETRY}

Laser Doppler flowmetry is a well established technique to characterise blood flow in vascularised tissues. The principles of laser Doppler flowmetry have been described in detail in the literature. ${ }^{5-7}$

The measurement of blood flow by laser light is based on the optical Doppler effect: laser light scattered by a moving particle is 
shifted in frequency by an amount $\Delta f$ as shown in equation (1).

$$
\Delta f=(1 / 2 \pi)\left(k_{\mathrm{sc}}-k_{\mathrm{i}}\right) \underline{\mathbf{v}}
$$

Where $k_{\text {sc }}$ is the wave vector of the scattered light, $k_{\mathrm{i}}$ is the wave vector of the incident light and $\underline{v}$ resembles the velocity vector of the moving particle. When the laser beam impinges on a tissue containing blood cells moving with different velocities in different directions, the spectrum broadens. It has a width that depends on the distribution of the velocity vectors and the scattering in the tissue itself. The collected light is guided to a photodetector and analysed using the method described by Bonner and Nosal. ${ }^{7}$

The 'effective Doppler shift' called 'flow' and the 'fraction power' of the Doppler shift, called 'volume' are calculated by the algorithm described in the appendix.

The mean RBC speed, called 'velocity', can be calculated by flow/velocity.

\section{Scanning laser Doppler flowmetry}

The so called SLDF method uses the ability of a laser scanning tomograph to measure the amount of backscattered light at different locations as the tissue of interest in a very short time. More detail of how the principles of Doppler flowmetry has been adapted for this application is given in the appendix.

\section{Reliability}

Five consecutive separate perfusion measurements of one eye were performed. Twelve eyes of 12 healthy persons were examined. The blood flow was measured at the same retinal area of each eye. To compare several measurements of the same retinal location in one eye the region of interest (ROI) was set in the vicinity of morphological landmarks of the retinal vasculature.

The reliability coefficient $r_{1}$ was calculated by the estimation of the variances of the biological component and the error component. The measured value is a combination of the true mean value, the biological variability component, and the error component (equation (2)). The variances of the two components are defined in equation (3). The error component was differentiated from the biological component by the variance component analysis calculated by the VAR COMP procedure from SAS-STAT (SAS STAT users guide release 6.03 edition. Cary, NC: SAS Institute Inc, 1988: 967-78).

$$
Y_{i j}=u+A_{i}+e_{i j}
$$

$Y_{i j}=$ measured value; $u=$ mean value; $A_{i}=$ biological component; $\mathrm{e}_{\mathrm{ij}}=$ measuring error component; $i=1, \ldots, n$ eyes; $j=1, \ldots, m$ measurements.

$$
\operatorname{Var}\left(\mathrm{Y}_{\mathrm{ij}}\right)=\operatorname{Var}\left(\mathrm{A}_{\mathrm{i}}\right)+\operatorname{Var}\left(\mathrm{e}_{\mathrm{ij}}\right)
$$

$\operatorname{Var}_{A}=$ variance of biological component; $\operatorname{Var}_{\mathrm{e}}=$ variance of error component.

The reliability coefficient $r_{1}$ is defined by the following equation:

$$
\mathrm{r}_{1}=\operatorname{Var}\left(\mathrm{Y}_{\mathrm{ij}}\right) /\left(\operatorname{Var}\left(\mathrm{Y}_{\mathrm{ij}}\right)+\operatorname{Var}\left(\mathrm{e}_{\mathrm{ij}}\right)\right)
$$

Validity

We verified the validity of the system by two techniques.

Firstly, the effect of changes in ocular pressure on flow and volume was studied. The ocular pressure was increased by a suction cup of $9 \mathrm{~mm}$ in diameter in three healthy volunteers. This method uses plastic suction cups of very low weight (about $0.2 \mathrm{~g}$ ) that adhere to the eye at negative pressures. The cup was placed on the temporal sclera of the eye. The vacuum was increased to negative pressures of 100,200 , and $300 \mathrm{~mm} \mathrm{Hg}$. The relation between the negative pressure applied and the resulting IOP was determined by available conversion curves. ${ }^{8}$ This curve was established for healthy volunteers of ocular refraction allowing deviations within +2.0 and $-2.0 \mathrm{dpt}$. After establishing the baseline by performing four perfusion pictures, the negative pressure was increased for 1 minute to $100 \mathrm{~mm} \mathrm{Hg}$, then to $200 \mathrm{~mm} \mathrm{Hg}$ and to $300 \mathrm{~mm} \mathrm{Hg}$. For each pressure level four perfusion pictures were performed. Retinal and optic nerve head blood flow were evaluated off line. The data of three retinal areas of $100 \mu \mathrm{m} \times 100 \mu \mathrm{m}$ were averaged.

Secondly, we proved how measurements of retinal blood flow performed by the presented method (SLDF) are correlated with data gained by a commercially available laser Doppler flowmeter (Oculix Inc). ${ }^{3}$ We examined the retinal blood flow in defined retinal areas by SLDF and by LDF (Oculix Inc) in 47 eyes. By plotting the measured blood flow variables. Flow, volume, and velocity of both devices the correlation coefficient and the $p$ value between SLDF and LDF were calculated.

In this preliminary study the reliability and the validity of the technique were estimated. Perfusion images of flow, volume, and velocity of the superficial retinal vasculature and of the deep ciliary sourced vasculature of the optic nerve head were investigated.

\section{Results}

\section{RELIABILITY}

The reliability of the quantitative analysis of the retinal perfusion map is good. The reliability coefficients $r_{1}$ of flow, volume, and velocity are $0.84,0.85$, and 0.84 respectively. This means that only $16 \%, 15 \%$, and $16 \%$ of the variability of the measurement of flow, volume, and velocity are produced by the error component.

\section{VALIDITY OF THE METHOD}

The retinal and optic nerve head blood flow decreases when increasing the negative pressure inside the suction cup. By increasing the negative pressure in the suction cup to $200 \mathrm{~mm} \mathrm{Hg}$ the retinal and optic nerve head blood flow decreased by $43 \%-54 \%$ and $34 \%-57 \%$ respectively, elevation of the negative pressure in the 
Figure 1 Retinal blood flow ('flow') measured by scanning laser Doppler flowmetry (SLDF) versus ocular perfusion pressure (PP) of three healthy volunteers. The IOP was increased by a suction cup. $P P$ was calculated by $P P=($ systolic arterial pressure + diastolic arterial pressure)/2-IOP. The IOP was changed by increasing the negative pressure in the suction cup to 100, 200, and $300 \mathrm{~mm} \mathrm{Hg}$. There is a significant linear relation between SLDF flow and

the ocular perfusion pressure $(\mathrm{r}=0.84$, $p<0.001)$.

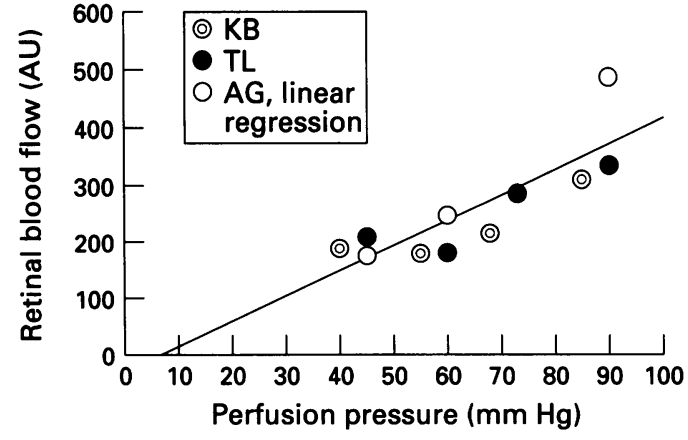

suction cup to $300 \mathrm{~mm} \mathrm{Hg}$ leads to diminished retinal and optic nerve head blood flow by $51 \%-69 \%$ and $52 \%-72 \%$ respectively. We found a significant linear relation $(r=0.84$, $p<0.001)$ between retinal blood flow and ocular perfusion pressure (Fig 1). Ocular perfusion pressure (PP) was calculated by the equation

\section{$\mathrm{PP}=$ (systolic $\mathrm{BP}+$ diastolic $\mathrm{BP}$ )/2-IOP}

Measurements of retinal blood flow by SLDF and a commercially available laser Doppler flowmetry system (Oculix Inc) of corresponding retinal points in 51 eyes showed significant linear correlations between flow $(r=0.83, \quad \mathrm{p}<0.0001)$, volume $\quad(r=0.51$, $\mathrm{p}<0.0001)$, and velocity $(r=0.59, \mathrm{p}<0.0001)$. In Figure 2 the measurements of flow in corresponding retinal areas performed by both methods in 51 eyes are depicted.

\section{QUALITATIVE AND QUANTITATIVE \\ EXAMINATION}

By the confocal technique perfused vessels and capillaries of the retinal superficial vasculature and of the deep ciliary sourced capillaries of the optic nerve head become visible. Figure 3 shows perfusion layers of the optic nerve head of different depths. Focusing on the superficial retina SLDF produces a picture of the superficial retinal vasculature of a normal eye (Fig 3, upper line). When focused deeper the superficial retinal capillaries disappear and only ciliary sourced capillaries of the prelaminar optic nerve head are depicted (Fig 3, lower lines).

Figure 4 demonstrates the retinal perfusion maps and averaged spectra of a $10 \times 10$ pixel area $(100 \mu \mathrm{m} \times 100 \mu \mathrm{m})$ of a normal eye and an

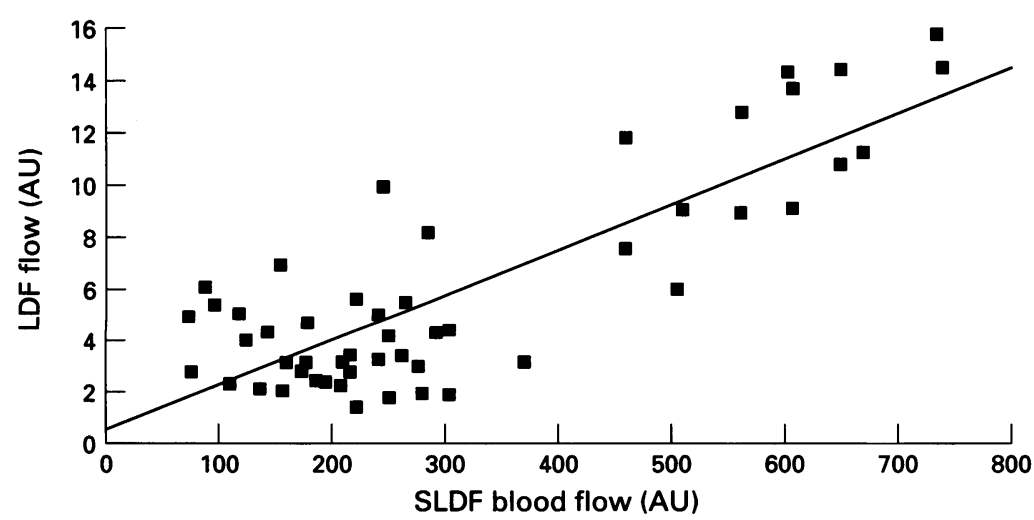

Figure 2 Relation between retinal blood flow measurements performed by scanning laser Doppler flowmetry (SLDF) and a commercially available laser Doppler flowmeter (Oculix Inc) of corresponding retinal areas in 51 eyes. There is a significant linear relation of flow $(\mathrm{r}=0.83, p<0 \cdot 0001)$ between both methods. eye with central retinal artery occlusion (CRAO). In the eye with CRAO the perfusion picture of the retina is 'dark' indicating reduced blood flow in retinal vessels and capillaries. Papillary capillaries sourced by ciliary arteries are bright resembling normal ciliary perfusion.

Using this technique quantitative measurements of the retinal blood flow become possible in any area of interest according to laser Doppler flowmetry. For any region of interest it is possible to express the power spectrum and the blood flow readings.

\section{Discussion}

To the best of our knowledge we present for the first time a non-invasive method capable of visualising the vasculature of the retina and the optic nerve head in a high definition.

The principle of blood flow measurement by SLDF is based on the scattering effect of the incident laser beam. Consequently this allows only blood flow quantification of the capillary meshwork. In contrast with the laser Doppler velocimetry of retinal arterioles, ${ }^{9}$ leading to absolute velocities in $\mathrm{mm} / \mathrm{s}$ by using two laser beams with defined angle, the method presented here produces blood flow readings in relative units. Riva et al showed that the Doppler broadening of capillary tissue in the optic nerve head and in the retina is maximally $300 \mathrm{~Hz}^{3}$ The technique presented here is able to measure Doppler broadening up to 2000 $\mathrm{Hz}$. Therefore, measurements of blood flow by this method in areas away from major vessels give readings of blood flow similar to commonly used laser Doppler techniques. As the blood velocity of larger vessels leads to Doppler broadening greater than $2000 \mathrm{~Hz}$, this technique is not capable of measuring blood flow in larger vessels. Only in regions of interest with Doppler broadenings lower than $2000 \mathrm{~Hz}$ were reliable measurements available.

The comparative measurements of retinal blood flow by SLDF and a commonly used laser Doppler flowmeter in corresponding retinal areas showed a significant and linear relation between SLDF and the commercially available laser Doppler flowmeter (Oculix).

Measurements of retinal and optic nerve head blood flow by SLDF showed that increased IOP is associated with decreased retinal blood flow. We found a significant linear relation $(r=0.84, \mathrm{p}<0.001)$ between retinal blood flow and ocular perfusion pressure.

These results indicate that SLDF is able to measure retinal blood flow in a similar way to the commercially available laser Doppler flowmeter.

The new method is non-invasive and uses a very low light intensity. Thus, multiple repetitions of measurements are possible. The resolution of $10 \mu \mathrm{m}$ of the scanning laser system leads to a visualisation of perfused retinal capillaries and vessels. By the confocal principle it is possible to focus the laser beam on deep layers in the prelaminar region of the optic nerve head and to visualise ciliary sourced capillaries nourishing the optic nerve head. The perfusion maps are very close to the 

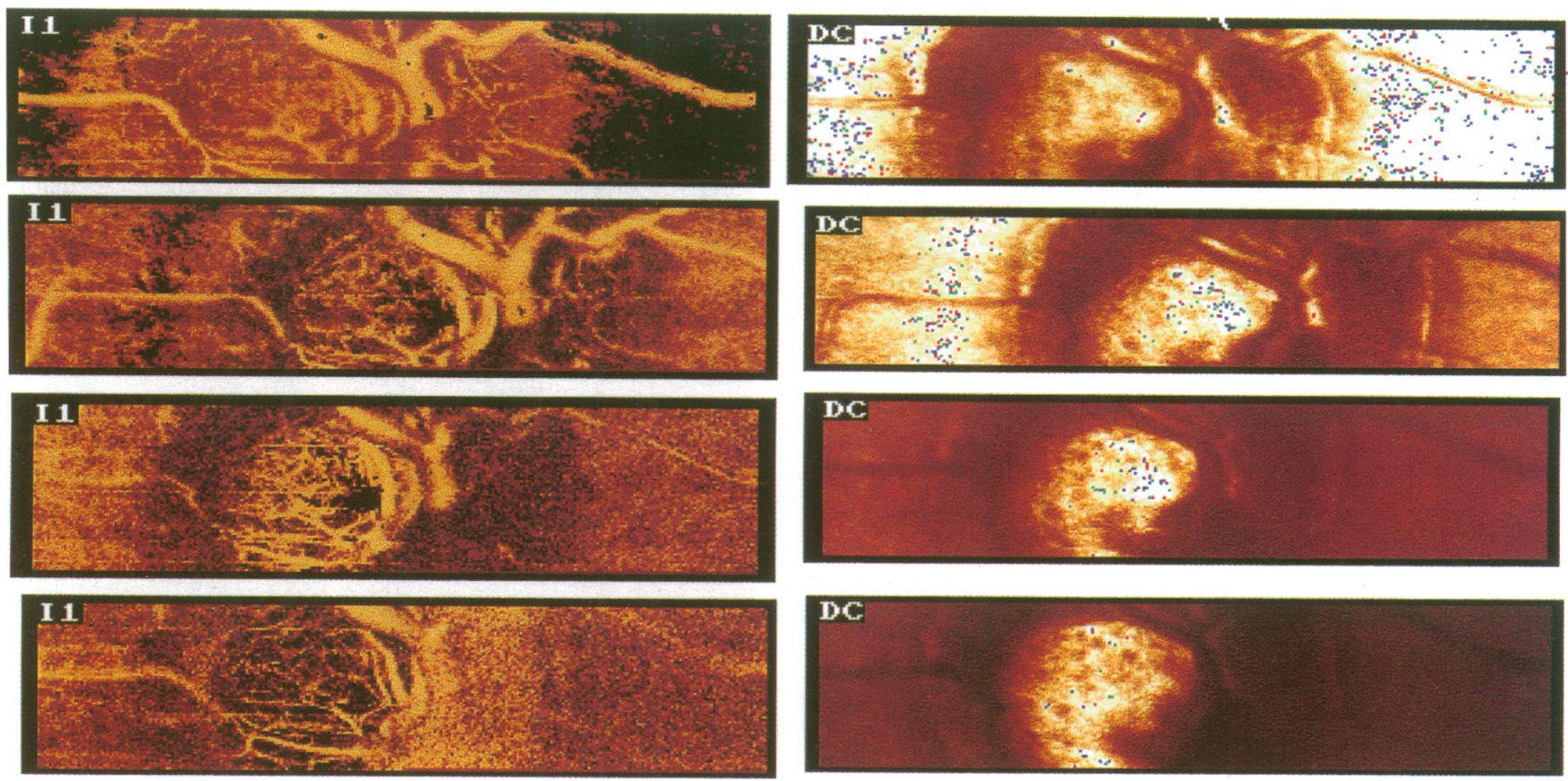

Figure 3 Tomography of normal optic nerve head. Left: scanning laser Doppler flowmetry. Right: ophthalmoscopy. Upper line: perfusion picture of a normal eye with the corresponding anatomical picture (DC), focused on the superficial retinal layer. The DC picture is equivalent to a retinal image which is performed by common laser scanning ophthalmoscopy. The perfusion picture shows finest blood vessels and retinal capillaries. Lower lines: perfusion picture of the same eye with the corresponding anatomical picture (DC), focused on the deeper layer in the optic nerve head. The layers of a depth of $300 \mu \mathrm{m}$ differ in depth by $90 \mu \mathrm{m}$. The perfusion picture shows ciliary sourced capillaries nourishing the prelaminar layer of the optic nerve head. Superficial retinal blood vessels and retinal capillaries are out of focus and invisible. As the retinal pigment epithelium absorbs the laser light with the wavelength used, choroidal vessels in the focused plane are not visible.
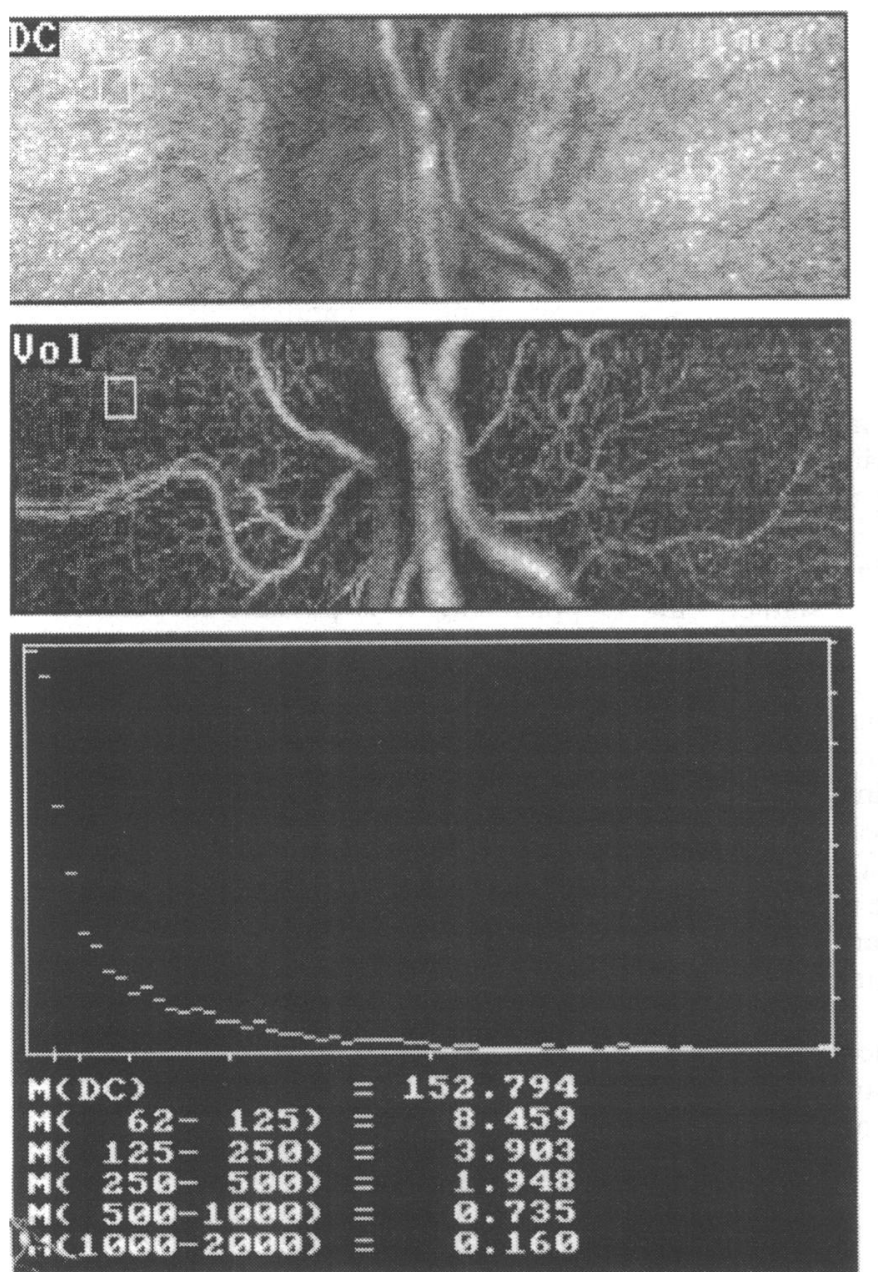
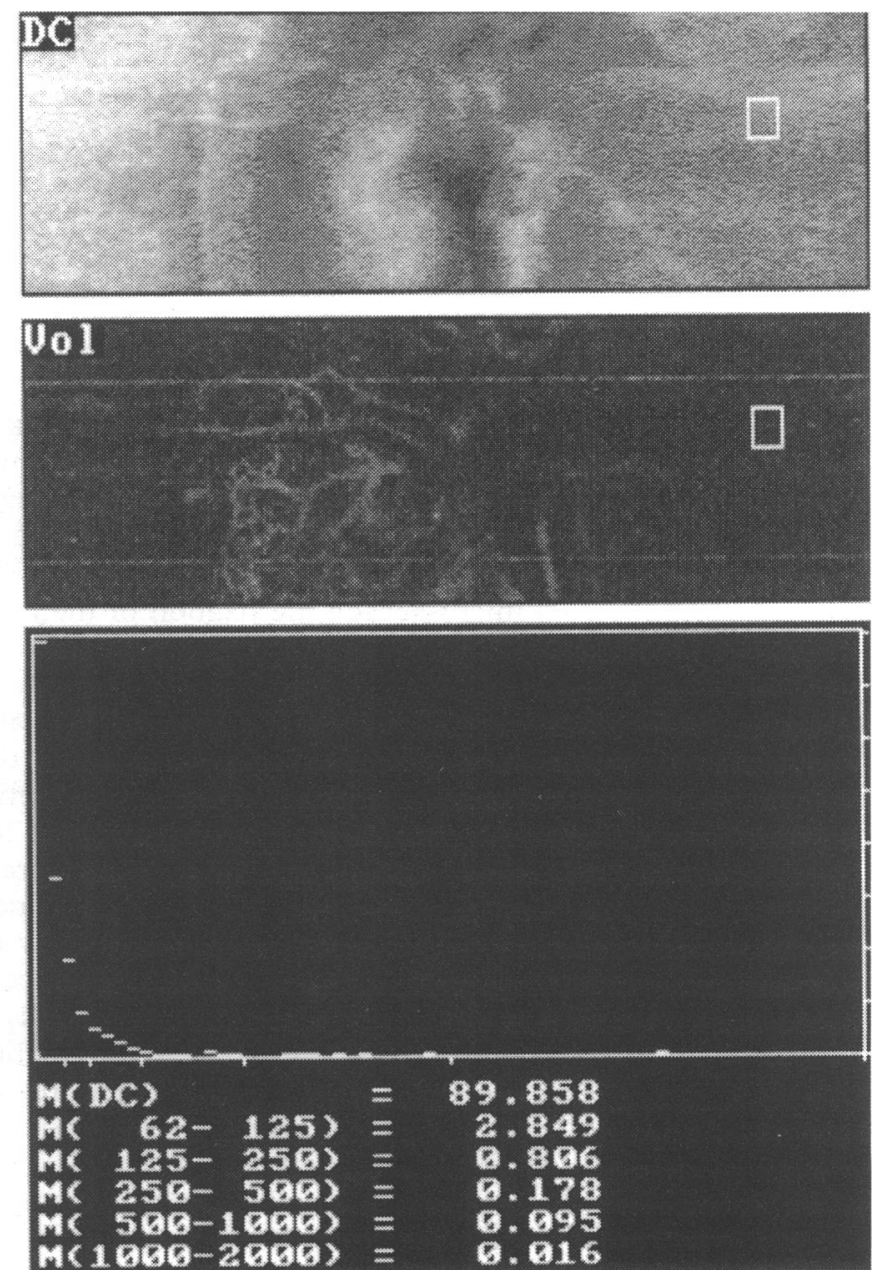

Figure 4 Retinal perfusion maps and averaged spectra of a $10 \times 10$ pixel area of a normal eye (left) and an eye with central retinal artery occlusion (CRAO) (right). Upper: anatomical pictures (DC image), performed by common laser scanning ophthalmoscopy. Middle: corresponding retinal perfusion maps. Bottom: the average spectra of the region of interest $(100 \mu \mathrm{m} \times 100 \mu \mathrm{m})$ expressed as frequency power diagram. The $x$-axis expresses the frequency in $\mathrm{Hz}$ from 0 to $2000 \mathrm{~Hz}$ in a logarithmic scale, the $y$-axis shows the 'power' in a linear scale. The readings $M(62-125), M(125-250), M(250-500)$, $M(500-1000), M(1000-2000)$ are the sums of power in the spectrum ranges from $66 \mathrm{~Hz} \mathrm{to} 125 \mathrm{~Hz}, 125 \mathrm{~Hz}$ to $250 \mathrm{~Hz}, 250 \mathrm{~Hz}$ to $500 \mathrm{~Hz}, 500 \mathrm{~Hz}$ to $1000 \mathrm{~Hz}$, and $1000 \mathrm{~Hz}$ to $2000 \mathrm{~Hz}$. The perfusion image of the eye with CRAO is dark indicating impaired retinal blood flow. In this eye the retinal perfusion is diminished compared with the normal eye in terms of laser Doppler flowmetry. The papillary vessels and capillaries sourced by ciliary arteries are bright resembling intact ciliary perfusion. 


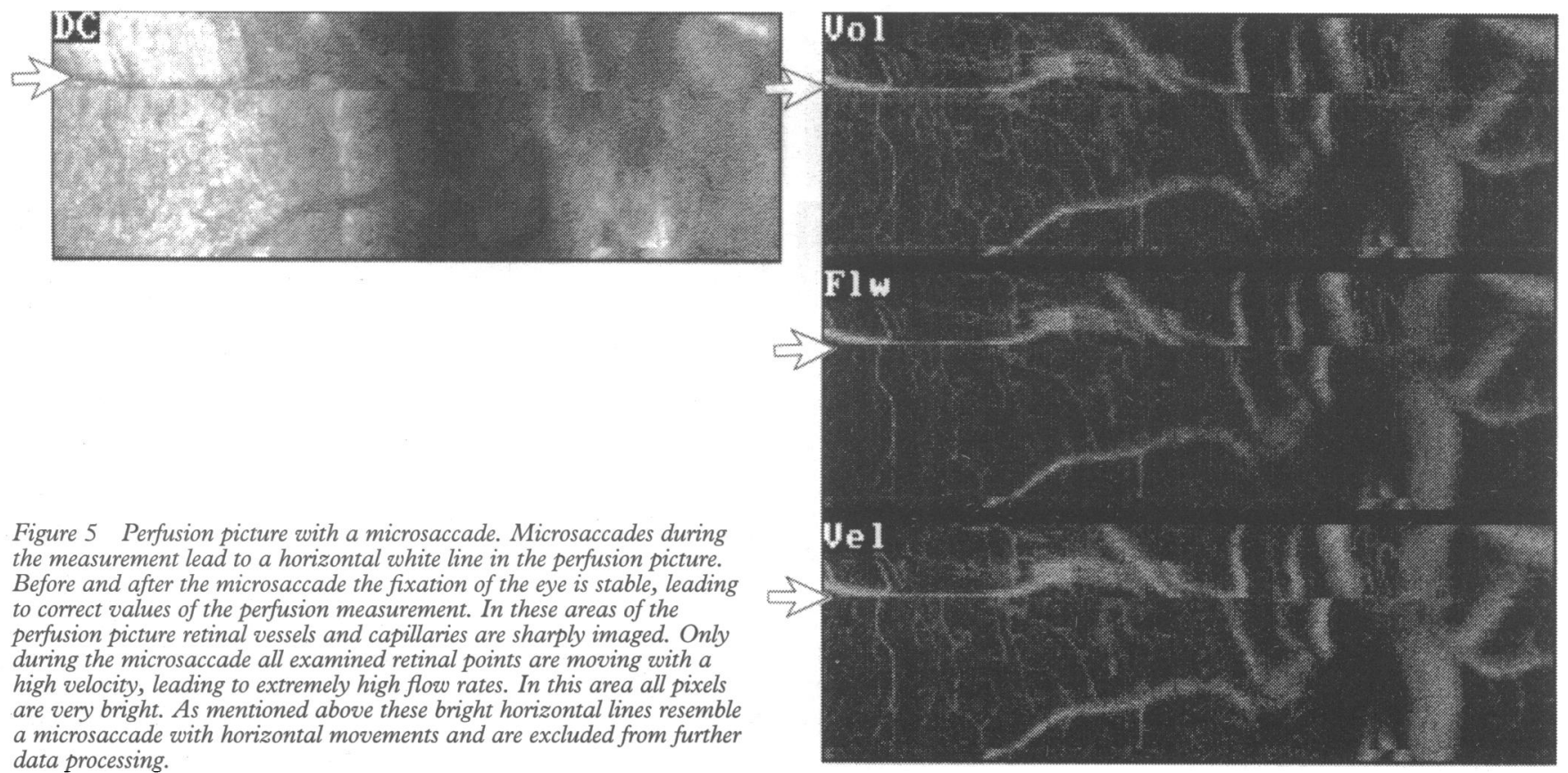

anatomical pictures performed by fluorescence angiography. It seems possible to visualise retinal areas of capillary non-perfusion by this method which is important in many diseases with altered retinal perfusion.

The good reliability may be due to the qualitative examination of the retinal perfusion images before using the perfusion images for blood flow quantification. Artificial movements or other disturbances are obviously visible in the perfusion pictures leading to a drop out of these perfusion pictures.

\section{LIMITATIONS OF THE METHOD}

The total time of measurement is 2 seconds. Therefore patients with bad fixation may show artefacts in the perfusion picture owing to eye movements (microsaccades) during the measurement, giving a horizontal white line in the perfusion picture. Before and after the microsaccade the fixation of the eye is stable, leading to correct values of the perfusion measurement. In these areas of the perfusion picture retinal vessels and capillaries are sharply imaged. Only during the time of the microsaccade are the examined retinal points moving with a high velocity, leading to extremely high flow rates. In this area all pixels are very bright. Thus, only the offline measurement of perfusion variables below and above the white line of the perfusion picture resembles correct perfusion values (Fig 5).

If the examined patient is not able to fixate at all, the perfusion picture is grey and shows no details of the retinal vasculature. In this case the picture is left out.

The choriocapillary meshwork is not imaged when focused on deep layers of the retina. Owing to the high absorption of the used laser light by the retinal pigment epithelium the choroidal vasculature remains invisible using laser light with wavelengths of 670 or $790 \mathrm{~nm}$.

The scattering processes of stationary and moving particles in retinal tissues can not be described exactly. Thus it is difficult to interpret the quantitative results for single measured pixels. The application of the Bonner and Nossal theory leads to impressive maps of the perfusion of the retina and the optic nerve head. The exact theory of the scattering processes (number of scattering events, scattering angles) needed to be investigated in more detail. Nevertheless, the described method yields a two dimensional index for retinal perfusion.

\section{Conclusion}

The combination of scanning laser ophthalmoscopy and laser Doppler flowmetry leads to a visualisation of perfused capillaries and vessels of the retina and the optic nerve head. SLDF enables a non-invasive, high resolution mapping of perfused vessels and capillaries of the retina and the optic nerve head. Using a two dimensional measurement of the perfusion coded by the Doppler shift it is possible to perform a reproducible quantification of capillary blood flow of distinct areas of the capillary meshwork of the retina and the optic nerve head.

The authors would like to thank Heidelberg Engineering and, particularly, Dr G Zinser for changing the construction of the laser scanning tomograph HE0815 and technical assistance in performing the experiments.

Supported by Deutsche Forschungsgemeinschaft Mi 320/23, Na 55/6-1.

1 Wolf S, Arend O, Toonen H, Bertram B, Jung F, Reim M Retinal capillary blood flow measurement with a scanning laser ophthalmoscope. Ophthalmology 1991; 98: 996 .

2 Stein MR, Parker CW. Reactions following intravenous fluorescein. Am f Ophthalmol 1971; 72: 861.

3 Riva CE, Harino S, Petrig BL, Shona RD. Laser Doppler flowmetry in the optic nerve. Exp Eye Res 1992; 55: 499.

4 Michelson G, Gründler A, Steinmeier R, Sigwanz U. Simultaneous measurement of ocular micro- and macrocirculation, intraocular pressure, and systemic functions. German f Ophthalmol 1994; 3: 48-53.

5 Ishimaru A. Theory and application of wave propagation and scattering in random media. Proc IEEE 1977; 65: 1030-61.

6 Maret G, Wolf PE. Multiple light scattering from disordered media. The effect of Brownian motion of ordered media. The effect of Brown.

7 Bonner RF, Nossal R. Principle of laser Doppler flowmetry. In: Sheperd Alber P, Oberg P Ake, eds. Laser Doppler blood flowmetry. Vol 107. Boston: Kluwer Academic Publishers, 1990: 17-45

8 Ulrich WD, Ulich C, Walther G. Ocular perfusion pressure and oculo-oscillo-dynamography. In: Lambrou GN, 
Greve EL, eds. Ocular blood flow in glaucoma. Amsterdam, Berkley, Milan: Kugler \& Ghedini Publications, 1989: 101-14.

9 Riva CE, Grunwald JE, Petrig BL. Laser Doppler measurement of retinal blood velocity: validity of the single scattering model. Applied Optics 1985; 24: 605

10 Europäisches Patent. Michelson Georg, Zinser Gerhard, Schmauß Bernhard: 'Verfahren und Gerät zur Messung der Fließgeschwindigkeit, insbesondere des Blutes. 1.6.94, European Patent No 94108430.3.

11 Elsner AE, ed. Topographic measurements at the fundus with the Heidelberg retina tomograph. In: Scanning laser ophthalmoscopy, tomography, and microscopy. New York: Plenum, 1993.

\section{Appendix}

PRINCIPLES OF FUNCTION OF SCANNING LASER DOPPLER FLOWMETRY

The so called scanning laser Doppler flowmetry method (SLDF) ${ }^{10}$ uses the ability of a laser scanning tomograph to measure the amount of backscattered light at different locations at the tissue of interest in a very short time. We used the scanning laser device of Heidelberg Engineering (Heidelberg). Since it is sufficient, in describing a fluctuating signal, to take samples from the signal with a sampling rate of at least twice the highest signal frequency, numerous points of the tissue can be examined very quickly by a scanning method.

For a single picture line the procedure is as follows: one sample from each point of the line is taken. After a flyback time this measurement is repeated several times for the same points with a high repetition rate. Thus, the backscattered intensities for each scanned point are obtained as a function of time (intensity-time curve). A spectrum analysis from the data of each location is performed using a fast Fourier transform algorithm. Thus the power spectrum of the Doppler shift of each retinal point is calculated by which the blood flow variables $\omega$ and $\chi$ can be computed. ${ }^{7}$

The 'effective Doppler shift' called 'flow' is calculated by equation (5).

$$
\omega=2 \pi\left(\sum_{125}^{2000 \mathrm{~Hz}} \mathrm{P}(\mathrm{f}) \mathrm{df}\right) / \mathrm{P}(\mathrm{f}=0)
$$

where $P(f) d f$ is the power of the photodetector current associated with fluctuations in a frequency range df about $f$ (in $\mathrm{Hz}$ ), and $P(f=0)$ is the DC power in the detected signal. 'Flow' describes the distance travelled by all moving cells inside the sample volume per unit time. Results independent of the reflectivity of the tested tissue are obtained by dividing the sum by the average power ( $=\mathrm{DC}$ power) received by the photodiode. Frequencies lower than $125 \mathrm{~Hz}$ were excluded to suppress the influence of mechanical vibrations.

The 'fraction power' of the Doppler shift, called 'volume' is calculated as

$$
x=2 \pi\left(\sum_{125}^{2000 \mathrm{~Hz}} \mathrm{f}(\mathrm{f}) \mathrm{df}\right) / \mathrm{P}(\mathrm{f}=0)
$$

Using equation (6) it is possible to calculate $\chi$, which is proportional to the mean number of photon collisions with moving cells. The value of $\chi$ can be used to determine the concentration of blood cells moving in the tissue. 'Volume' is proportional to the number of moving red blood cells (RBCs) in a sample volume of tissue.

The mean RBC speed, called 'velocity' can be calculated from equation (7).

$$
\text { Velocity }=\omega / \chi
$$

Performing this sampling procedure point by point yields the map of the blood perfusion of a distinct retinal area. Our data acquisition and evaluation system is a modified laser scanning tomograph. The spatial resolution accuracy of the scanning laser system is 10 $\mu \mathrm{m} .{ }^{11}$ The examined retinal area has a size of $2.7 \mathrm{~mm} \times 0.7 \mathrm{~mm}$ and is mapped by a resolution of 256 points $\times 64$ lines. Laser diodes with wavelengths of $670 \mathrm{~nm}$ and $790 \mathrm{~nm}$, respectively, and optical powers of $100 \mu \mathrm{W}$ and $200 \mu \mathrm{W}$, respectively, are used as the light source. Laser light with a wavelength of $670 \mathrm{~nm}$ is subjectively brighter for the patient than $790 \mathrm{~nm}$. On the other hand, laser light at $790 \mathrm{~nm}$ produces better tomographic pictures of the optic nerve head vasculature by better penetration through the neuronal tissue.

The scanning laser system examines the retina in a $10^{\circ}$ field without pupil dilatation using a confocal optical system. Each horizontal line of 256 pixels is scanned 128 times with a line repetition rate of $4000 \mathrm{~Hz}$. This process is repeated for each of the 64 horizontal rows. This leads to an intensity matrix of 256 points $\times 128$ times $\times 64$ lines. The total data acquisition time is 2.048 seconds. Performing a fast Fourier transform (FFT) of the 128 intensity values of each retinal point yields the spectrum of the Doppler shift of any retinal point. Doppler frequencies less than $2000 \mathrm{~Hz}$ can be detected correctly using a line sampling frequency of $4000 \mathrm{~Hz}$. To minimise the influence of other movements such as breathing, cardiac action, and intensity fluctuation owing to other effects frequencies lower $125 \mathrm{~Hz}$ were excluded.

With the calculated data of flow (equation (5)), volume (equation (6)), and velocity (equation (7)) two dimensional maps of the flow, volume, or velocity are created where the brightness of each pixel encodes the movement of the blood cells in the corresponding retinal area. The brighter the pixel the faster the cells move at this retinal point. For quantification of capillary retinal blood flow in terms of laser Doppler flowmetry in relative units the mean value and the histogram of flow, volume, and velocity may be calculated in any region of interest of the retinal perfusion picture. Figure 6 shows schematically the principle of function of SLDF.

By the confocal principle of the scanning laser system used it is possible to focus the laser beam on superficial or deep layers in the prelaminar region of the optic nerve head. The thickness of an imaged layer is $300 \mu \mathrm{m}$, owing to the optical characteristics of the eye and the SLDF machine (optical resolution parallel to the optical axis of the eye, size of the optical aperture, etc). Thus only light which comes from the imaged layer of $300 \mu \mathrm{m}$ is detectable. 

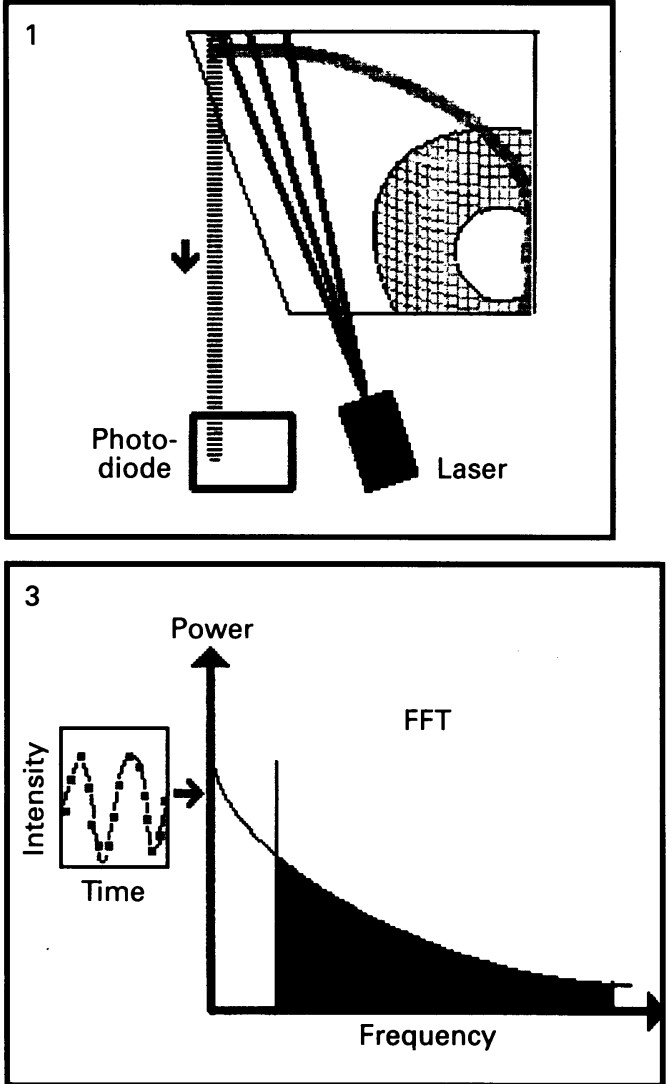

Figure 6 Scheme of the principle of scanning laser Doppler flowmetry (FFT=fast Fourier transformation).

\section{Technical settings}

To get optimal pictures some technical settings were necessary. When the laser beam was focused on the superficial retinal vessels, the real time reflectivity picture showed sharply focused details of retinal vessels and capillaries. In case of opacities of the cornea, lens, or vitreous the reflectivity as well as the perfusion image showed no details. During the measurement time of 2 seconds the patient had to fixate with the fellow eye a small point in a distance of 2 metres. After calculating the Doppler broadening of each pixel by the fast Fourier transformation the perfusion images of flow, volume, and velocity were built. In any region of interest all perfusion variables were available (average power spectrum, histogram of flow, volume, and velocity, mean (standard deviation) of flow, volume, and velocity, $\mathrm{P}(\mathrm{f}=0)$ [representing the reflectivity ( $=\mathrm{DC}$ value)]. The size of the region of interest (ROI) is variable (from 1 pixel $\times 1$ pixel to 50 pixels $\times 50$ pixels). The optimal size was 10 pixels $\times 10$ pixels $(=100 \mu \mathrm{m} \times 100 \mu \mathrm{m})$. The operator

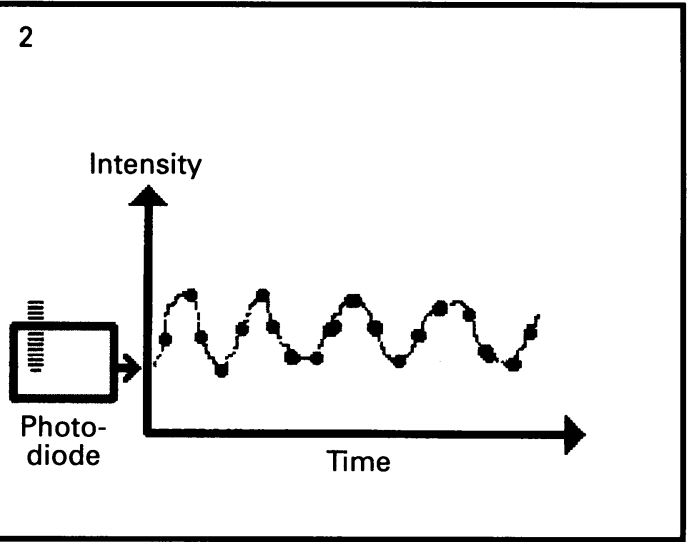

4

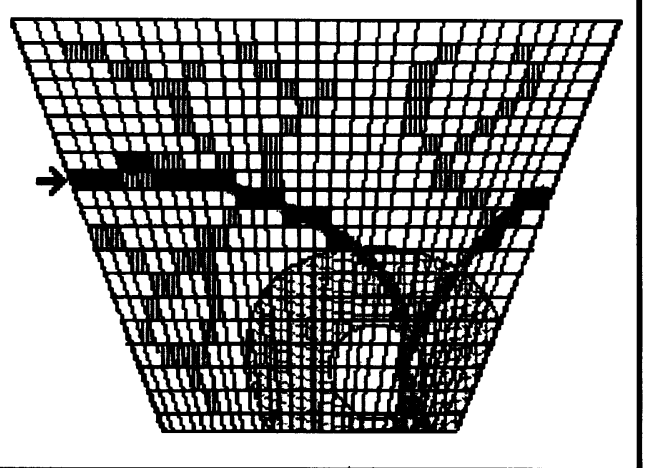

identified the $\mathrm{ROI}$ in the perfusion picture by 'mouse'. Using the algorithm the perfusion variables were normalised regarding the reflectivity by division through $\mathrm{P}(\mathrm{f}=0)$ (see equations (5) and (6)). Despite this, the DC value of the ROI should be in the range of 80 and 150 arbitrary units.

Reliable measurements of retinal perfusion variables were only available in ROIs without larger vessels. Riva et al showed that the Doppler broadening of capillary tissue in the optic nerve head and in the retina is maximally $300 \mathrm{~Hz}^{3}$ The technique presented can measure Doppler broadening up to $2000 \mathrm{~Hz}$. As the blood velocity of larger vessels leads to Doppler broadening greater than $2000 \mathrm{~Hz}$, this technique is not able to measure blood flow in larger vessels. Only in regions of interest with Dopper broadenings lower than $2000 \mathrm{~Hz}$ were reliable measurements available. Therefore measurements of blood flow by the method presented in areas away from major vessels give readings of blood flow similar to commonly used laser Doppler techniques. 